\title{
Optimizing geometric measures for fixed minimal annulus and inradius
}

\author{
María A. Hernández Cifre and Pedro J. Herrero Piñeyro
}

\begin{abstract}
In this paper we relate the minimal annulus of a planar convex body $K$ with its inradius, obtaining all the upper and lower bounds, in terms of these quantities, for the classic geometric measures associated with the set: area, perimeter, diameter, minimal width and circumradius. We prove the optimal inequalities for each one of those problems, determining also its corresponding extremal sets.
\end{abstract}

\section{Introduction}

Let $K$ be a convex body (compact convex set) in the Euclidean plane. Associated with $K$ there are a number of well-known functionals: the area $A=A(K)$ and the perimeter $p=p(K)$; the diameter $D=D(K)$ and the minimal width $\omega=\omega(K)$ (minimum distance between two parallel support hyperplanes of $K$ ); among all discs containing $K$ there is exactly one with minimum radius, called the circumradius $R_{K}$ of the set $K$; respectively, among all discs which are contained in $K$, those whose radii have maximum value, provide the inradius of the body, $r_{K}$. These special discs (named circumcircle and incircles) have very useful properties; some of them will be stated and used later.

Another interesting functional to be considered for a convex body $K$ is the thickness of its minimal annulus. The minimal annulus of $K$ is the annulus (the closed set consisting of the points lying between two concentric discs - concentric $n$-balls in $\mathbb{R}^{n}$ ) with minimal difference of radii that contains the boundary of $K$. Of course, the minimal annulus is uniquely determined (Bonnesen, [2], in $\mathbb{R}^{2}$ and $\mathbb{R}^{3}$, and Bárány, [1], in higher dimension). From now on, we shall denote by $A(c, r, R)$ the minimal annulus of the planar

2000 Mathematics Subject Classification: 52A40, 52A10, 52A38.

Keywords: Convex bodies, minimal annulus, inradius, area, perimeter, circumradius, diameter, minimal width. 
convex body $K$, where $c, r$ and $R$ represent, respectively, its center, radius of the inner circle, and radius of the outer circle. This object and its properties were studied mainly by Bonnesen for planar convex sets (see [2] and [3]). More recently, very interesting works have appeared, in which, the minimal annulus has been studied in a more general setting: for arbitrary dimension, replacing the ball by the boundary of a fixed smooth strictly convex body, in Minkowski space... (see, for instance, $[1,8,9,10,11,14]$ ).

Another interesting problem would be to look for inequalities involving the classical functionals and the minimal annulus, finding the convex sets for which the equality sign is attained: the extremal sets. In [2], [5] and [4], Bonnesen and Favard studied this type of problems: in [2] and [5] the minimum and the maximum of the isoperimetric deficit $p^{2} /(4 \pi)-A$ were obtained; in the third paper, the optimal bounds of the area and the perimeter for fixed minimal annulus were determined.

In [6], the bounds for the remaining measures (diameter, minimal width, circumradius and inradius) in terms of the minimal annulus have been obtained, as well as the optimal inequalities that state the best bounds for the classical magnitudes when the minimal annulus and the circumradius are fixed (see [6] and [7]): let us note that if three measures are involved, the question becomes more interesting when the equality, for a particular inequality, is not attained for a single figure, but for a continuous family of sets; in this case, that inequality, named optimal, provides the maximum or minimum value of a measure for each pair of possible values of the others.

In this paper, we obtain all the possible (and optimal) relations which state the maximum and minimum values of the area, the perimeter, the diameter and the minimal width of a convex body, when its minimal annulus and its inradius are given. We prove the optimal inequalities for each one of these problems, determining also their corresponding extremal sets. The circumradius case was solved in [7].

\section{Some previous results}

Before stating the main results of the paper, let us consider some properties of the minimal annulus of a convex body $K$, which will play a crucial role in the proofs of the results. Let us denote by $c_{r}$ and $C_{R}$, respectively, the inner and the outer circles of the minimal annulus $A(c, r, R)$ of $K$.

As usual, $\partial K$ will denote the boundary of the set $K$. Given two points $P, Q \in \mathbb{R}^{2}, P Q$ will denote the straight line determined by them; $\overline{P Q}$ the line segment joining them; and $\overparen{P Q}$ any circular arc with $P, Q$ as extreme points. Besides, if $P, Q$ lie on a circumference (with center $c$ ), we call central angle of $P$ and $Q$ the angle $\angle(P c Q)$ determined by them with respect to the center $c$. 
The following well-known properties were studied by Bonnesen in [2]:

(P1) Each one of the circumferences $\partial c_{r}$ and $\partial C_{R}$ touches the boundary $\partial K$ of $K$ in, at least, two points.

(P2) The sets $\partial c_{r} \cap \partial K$ and $\partial C_{R} \cap \partial K$ can not be separated.

(Two sets $A$ and $B$ can be separated if there exists a line $\ell$ such that $A \subset \ell^{+}$and $B \subset \ell^{-}$, where $\ell^{+}, \ell^{-}$represent the halfplanes determined by $\ell$ ).

(P3) The minimal annulus of a convex body $K$ is uniquely determined.

(P4) The minimal annulus of a convex body $K$ is the only annulus that contains $\partial K$ and verifies properties $(\mathbf{P 1})$ and (P2).

The following lemmas were obtained in [6], where we proved some properties of the minimal annulus of a convex body $K$, as well as its relation with the inradius of $K$. They will be very useful in the proofs of the results.

Lemma 1. Let $K$ be a convex body with minimal annulus $A(c, r, R)$. The following properties hold:

(a) There are points $P, Q \in \partial C_{R} \cap \partial K$ whose central angle $\alpha$ verifies $\alpha \geq 2 \arccos (r / R)$.

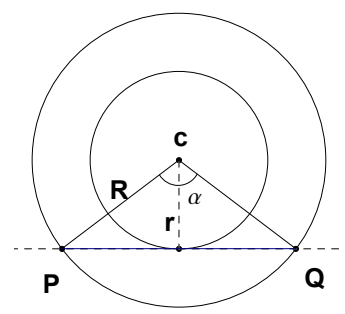

Figure 1. The limit case when the central angle of the points $P, Q \in \partial C_{R} \cap \partial K$ is, precisely, $\alpha=$ $2 \arccos (r / R)$.

(b) $K$ contains a 2-cap-body generated by the convex hull of $c_{r}$ and two points of $\partial C_{R} \cap \partial K$, whose minimal annulus is $A(c, r, R)$ (a cap-body is the convex hull of a disc and countable many points such that the line segment joining any pair of those points intersects the disc).

(c) $K$ is contained in a circular slice of the outer circle $C_{R}$ determined by two support lines to $c_{r}$, whose minimal annulus is $A(c, r, R) \quad(a$ circular slice is the part of a circle bounded by two straight lines, whose intersection point, if it exists, is not interior to it).

The following lemma collects some properties relating the minimal annulus of a convex body with its inradius. From now on, we shall denote by $c_{K}$ an incircle of the body $K$, and by $y_{0}$ one of its incenters. 
Lemma 2. Let $K$ be a convex body with minimal annulus $A(c, r, R)$ and incircle $c_{K}$. The following properties hold:

(i) $r \leq r_{K}$.

(ii) $\operatorname{conv}\left(c_{r} \cup c_{K}\right) \subset K \subset C_{R}$.

(iii) $c_{r}$ can not be strictly contained in $c_{K}$, having the following possible relative positions between them (see Figure 2):

(a) $c_{r} \equiv c_{K}$.

(b) $\partial c_{r} \cap \partial c_{K}$ contains, exactly, two points.

(c) The boundaries $\partial c_{r}, \partial c_{K}$ touch (from outside) in one point.

(d) There are no common points.

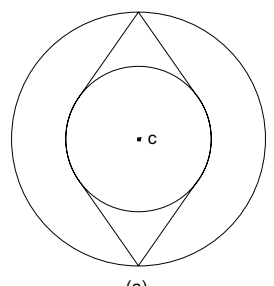

(a)

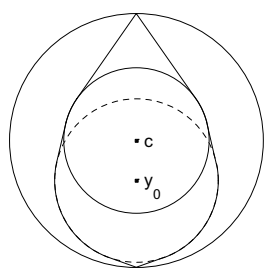

(b)

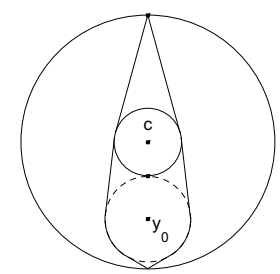

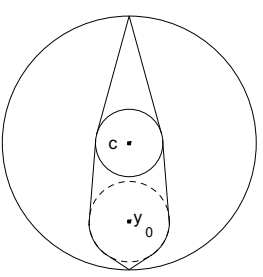

Figure 2: Some examples for the relative positions of $c_{r}$ and $c_{K}$.

(iv) The boundary of the set $\operatorname{conv}\left(c_{r} \cup c_{K}\right)$ is formed by two line segments $\overline{P S}$ and $\overline{Q T}$, and the corresponding circular arcs $\overparen{P Q} \subset \partial c_{r}$ and $\overparen{S T} \subset \partial c_{K}$. Then, each of those arcs has, at least, one point of $\partial K$.

(v) The arc $\overparen{P Q} \subset \partial c_{r}$ contains two points $P^{\prime}, Q^{\prime} \in \partial K$ (which can coincide with $P$ and $Q)$, determining a central angle $\alpha \geq 2 \arccos (r / R)$.

Let us add a new property to this lemma which will be needed also later:

(vi) $K$ contains a convex body $K^{2 c}=\operatorname{conv}\left\{c_{r} \cup c_{K}, N, M\right\}$, with the same minimal annulus and inradius as $K$, where $N$ and $M$ lie, respectively, on each arc of $\partial C_{R}$ determined by the lines $P S$ and $Q T$ (see figure 3).

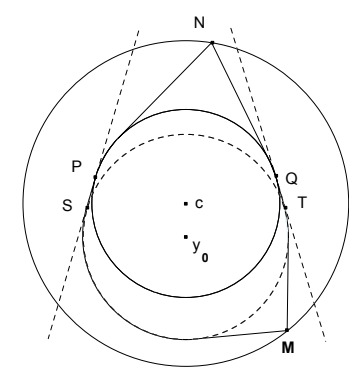

Figure 3: A set $K^{2 c} \subset K$ with the same minimal annulus and inradius. 
Proof of property (vi): Property (v) of lemma 2 assures the existence of, at least, two points $P^{\prime}, Q^{\prime}$ of $\partial K$, lying on the circular arc $\overparen{P Q} \subset \partial c_{r}$, and determining a central angle $\alpha \geq 2 \arccos (r / R)$. Then, $K$ is contained in the circular slice $K^{s}$ of $C_{R}$ determined by the support lines to $c_{r}$ through $P^{\prime}$ and $Q^{\prime}$ (see figure 4).

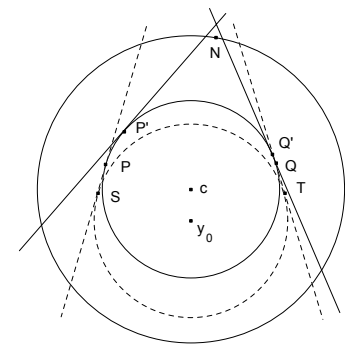

(a)

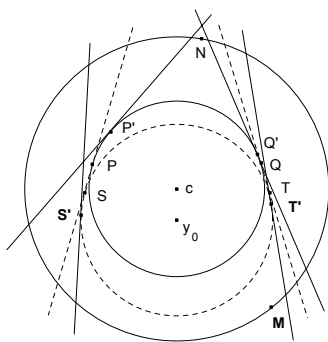

(b)

Figure 4: Construction of the set $K^{2 c} \subset K$.

On the other hand, property (b) of lemma 1 states that $K$ contains a 2-cap-body $K^{c}=\operatorname{conv}\left\{c_{r}, N, M\right\}$, with $N, M \in \partial C_{R} \cap \partial K$. Besides, this cap-body can be chosen in such a way that there is one vertex lying on each circular arc of the boundary of $K^{s}$; in the opposite case, property (P2) would be contradicted. Hence, we can suppose, for instance, that $N$ lies on the circular arc of $\partial K^{s}$ which is "closer" to $\overparen{P^{\prime} Q^{\prime}}$ (see figure $4(\mathrm{a})$ ), and $M$ on the opposite arc.

Now, since $c_{K}$ is an incircle of $K$, it meets $\partial K$ either in two diametrically opposite points, or in three points that form the vertices of an acute-angled triangle (see [3]); or equivalently, in three points which do not lie on the same half-circumference. Thus, we can always choose two points $S^{\prime}, T^{\prime} \in \partial c_{K} \cap \partial K$ in such a way that $\overparen{S^{\prime} T^{\prime}} \subset \overparen{S T}$ is bigger than a half-circumference. It implies that the support lines to $K$ through $S^{\prime}$ and $T^{\prime}$ do not intersect in the interior of $C_{R}$, and they determine a new circular arc on which the point $M$ lies (see figure 4(b)). Let us note that the points $P^{\prime}, Q^{\prime}, S^{\prime}, T^{\prime}$ can coincide with $P, Q, S, T$, respectively. So, $K$ contains the set $K^{2 c}=\operatorname{conv}\left\{c_{r} \cup c_{K}, N, M\right\}$, with $N, M$ verifying the assumptions of the result.

Finally, let us note that $K^{2 c}$ has minimal annulus $A(c, r, R)$ by property (P4) and inradius $r_{K}$, since its boundary contains, necessarily, diametrically opposite points of $\partial c_{K}$.

In the following, we are going to obtain all the possible (and optimal) relations which state the maximum and minimum values of the area, the perimeter, the diameter and the minimal width of a convex body, when its minimal annulus and its inradius are given. The circumradius case was studied and solved in [7]. 


\section{Optimizing the area and the perimeter}

In this section we state the relation between the minimal annulus, the inradius and both, the area and the perimeter of a convex body $K$. More precisely, we are going to obtain the best bounds (upper and lower bounds) for $A$ and $p$, when we suppose that the minimal annulus of the convex body and its inradius are fixed, determining also the extremal sets in each case. We start with the upper bounds.

Let us recall that, by lemma 1(c), if the minimal annulus of $K$ is $A(c, r, R)$, then $K$ is contained in a circular slice $K^{s}$ of $C_{R}$ determined by support lines to $c_{r}$ through two points of $\partial c_{r} \cap \partial K$; all these sets have the same area and the same perimeter, and thus, it holds

$$
\begin{aligned}
& A \leq A\left(K^{s}\right)=2\left(r \sqrt{R^{2}-r^{2}}+R^{2} \arcsin \frac{r}{R}\right) \\
& p \leq p\left(K^{s}\right)=4\left(\sqrt{R^{2}-r^{2}}+R \arcsin \frac{r}{R}\right) .
\end{aligned}
$$

The following theorem states also these ones as the upper bounds for any value $r_{K}$ of the inradius.

Proposition 1. Let $K$ be a convex body with minimal annulus $A(c, r, R)$ and inradius $r_{K}$. Then,

$$
\begin{aligned}
& A \leq 2\left(r \sqrt{R^{2}-r^{2}}+R^{2} \arcsin \frac{r}{R}\right), \\
& p \leq 4\left(\sqrt{R^{2}-r^{2}}+R \arcsin \frac{r}{R}\right) .
\end{aligned}
$$

The equality holds, in both inequalities, if and only if the convex body $K$ is the circular slice of $C_{R}$ determined by the common support lines to $c_{r}$ and $c_{K}$, when $\partial c_{K}$ touches (in the interior) $\partial C_{R}$ (see figure 5).
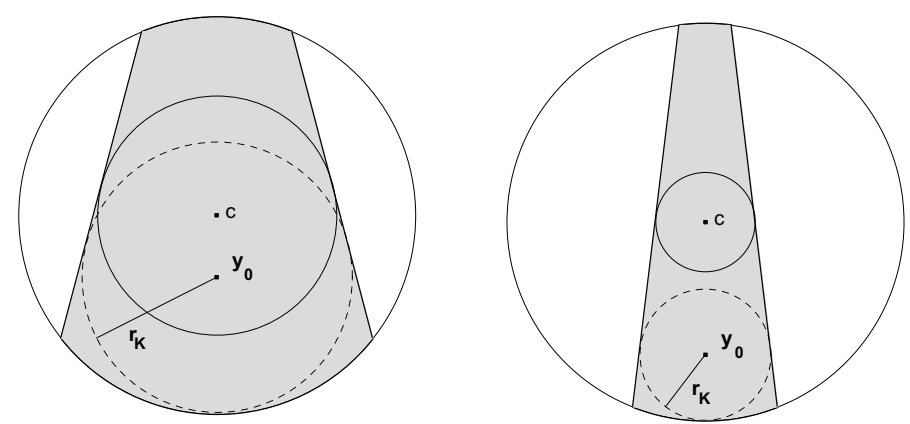

FiguRE 5: Circular slices of $C_{R}$ with maximum area and perimeter. 
Proof: We just have to see that, if $r_{K}$ is the inradius of $K$, then there is a circular slice $K^{s}$ of $C_{R}$ (verifying the above assumptions) with inradius $r_{K}$. In order to do that, let us take the incenter $y_{0}$ in such a way that $\partial c_{K}$ touches $\partial C_{R}$. Then, the circular slice $K^{s}$ of $C_{R}$ determined by the common support lines to $c_{r}$ and $c_{K}$ has inradius $r_{K}$, since $\partial c_{K}$ contains three points that form an acute-angled triangle (see figure 5 ); and by minimal annulus $A(c, r, R)$ by property $(\mathbf{P} 4)$. Let us note that the convex body $K^{s}$ so generated is always a circular slice, i.e., the lines determining it do never intersect in the interior of $C_{R}$; it holds because $r_{K} \leq 2 R r /(R+r)$ always (see [6, Subsect. 3.4, Prop. 7]), and just for the equality case the intersection point lies on the boundary of $C_{R}$.

We conclude this section stating the lower bounds for the area and the perimeter of a convex body with prescribed minimal annulus and inradius.

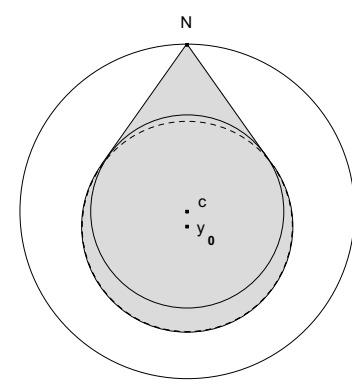

Figure 6 . The set $K_{d}$.

We state some useful notation: for $r_{K}$ and $A(c, r, R)$ given, let us suppose the incenter $y_{0}$ located to the suitable distance of $c$ in order to the intersection point of the common support lines to $c_{r}$ and $c_{K}$ lies, precisely, on the boundary of $C_{R}$; this point will be denoted by $N$ (see figure 6). It is an easy computation to check that this distance is given by $d\left(y_{0}, c\right)=R\left(r_{K}-r\right) / r$. We are going to denote by $K_{d}:=\operatorname{conv}\left\{c_{r} \cup c_{K}, N\right\}$ (see figure 6), which is just the cap-body $\operatorname{conv}\left\{c_{K}, N\right\}$, since it always holds the relation $r_{K} \geq r$.

Theorem 1. Let $K$ be a convex body with minimal annulus $A(c, r, R)$ and inradius $r_{K}$. Then,

$$
\begin{aligned}
& A \geq \frac{r_{K}^{2}}{r}\left(\sqrt{R^{2}-r^{2}}+\frac{1}{r_{K}} \sqrt{R^{2}\left(2 r-r_{K}\right)^{2}-r^{2} r_{K}^{2}}\right)+r_{K}^{2} \theta, \\
& p \geq 2\left(\frac{r_{K}}{r} \sqrt{R^{2}-r^{2}}+\frac{1}{r} \sqrt{R^{2}\left(2 r-r_{K}\right)^{2}-r^{2} r_{K}^{2}}+r_{K} \theta\right),
\end{aligned}
$$

where

$$
\theta=\arcsin \frac{r r_{K}}{R\left(2 r-r_{K}\right)}+\arcsin \frac{r}{R} .
$$

The equality holds, in both inequalities, if, and only if, $K=\operatorname{conv}\left\{K_{d}, M\right\}$, where $M$ is the point of $\partial C_{R}$ diametrically opposite to $N$ (see figure 7). 


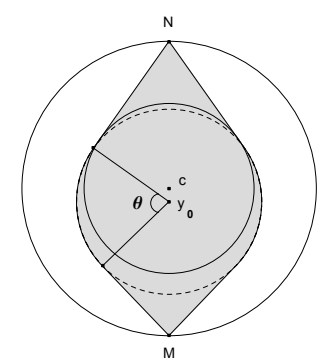

FiguRE 7: The convex body $\operatorname{conv}\left\{K_{d}, M\right\}$ has minimum area and perimeter.

Proof: Let us notice that, when $r_{K}=r$, inequalities (3.3) and (3.4) turn into, respectively, the well-known relations

$$
A \geq 2 r\left(\sqrt{R^{2}-r^{2}}+r \arcsin \frac{r}{R}\right) \quad \text { and } \quad p \geq 4\left(\sqrt{R^{2}-r^{2}}+r \arcsin \frac{r}{R}\right)
$$

(see [4]); in both cases, the extremal set is a cap-body with two vertices lying on $\partial C_{R}$. Thus, from now on we will assume that $r_{K}>r$ and hence, that $c_{K} \not \equiv c_{r}$.

We know (lemma 2(vi)) that $K$ contains a set $K^{2 c}=\operatorname{conv}\left\{c_{r} \cup c_{K}, N, M\right\}$, for suitable $N, M \in \partial C_{R}$ (see figure 8(a)), and therefore, $A \geq A\left(K^{2 c}\right)$ and $p \geq p\left(K^{2 c}\right)$. Since $K^{2 c}$ has minimal annulus $A(c, r, R)$ and inradius $r_{K}$, we have reduce the problem to study the area and the perimeter for this particular family of sets.

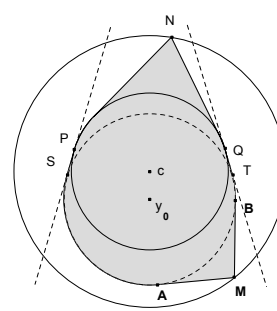

(a)

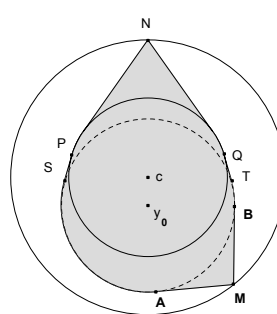

(b)

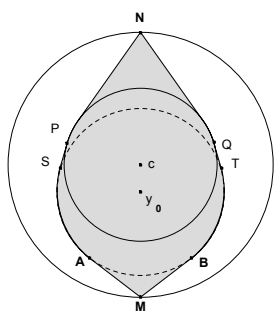

(c)

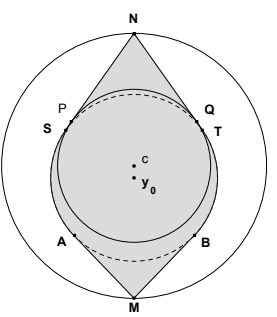

(d)

Figure 8: Minimizing the area and the perimeter.

Let us note first that, for each fixed $y_{0}$, the area and the perimeter of the set $\operatorname{conv}\left\{c_{r} \cup c_{K}, N\right\}$ are constant for any possible position of $N$ (it moves just on the arc of $\partial C_{R}$ determined by the support lines to $\partial c_{r}$ through $P$ and $Q$, see figure $8(\mathrm{a}))$, due to $c_{r}$ and $C_{R}$ are concentric. Thus, without loss of generality, we can choose $N$ to be the intersection point of the line $c y_{0}$ with $\partial C_{R}$ (see figure $8(\mathrm{~b})$ ).

On the other hand, if we denote by $A$ and $B$ the tangent points where the support lines to $c_{K}$ (which passes through $M$ ) touch $\partial c_{K}$, the set limited by the circular arc $\overparen{A B}$ and the line segments $\overline{A M}$ and $\overline{B M}$, has minimum area 
when the distance from $M$ to $\partial c_{K}$ is as small as possible; this is, when $M$ is the second intersection point of the straight line $c y_{0}$ with $\partial C_{R}$. It is also in this case when the length of the line segments $\overline{A M}$ and $\overline{B M}$ is the smallest possible (see figure $8(\mathrm{c})$ ). So, it suffices to consider the sets $K^{2 c}$ which are symmetric with respect to the line $c y_{0}$.

Let us denote by $x$ the distance between the centers $y_{0}$ and $c, x:=$ $d\left(y_{0}, c\right)$. It is clear that

$$
\frac{R}{r}\left(r_{K}-r\right) \leq x \leq R-r_{K},
$$

where the lower bound corresponds to the limit case when the common support lines to $c_{r}$ and $c_{K}$ intersect precisely on $N$, whereas the upper bound is given when $\partial c_{K}$ touches $\partial C_{R}$. It is a tedious calculation to compute the area and the perimeter of these figures in terms of the distance $x$ :

$$
\begin{aligned}
A(x)= & \left(r+r_{K}\right) \sqrt{x^{2}-\left(r_{K}-r\right)^{2}}+r_{K} \sqrt{(R-x)^{2}-r_{K}^{2}}+r \sqrt{R^{2}-r^{2}} \\
& +\left(r_{K}^{2}-r^{2}\right) \arcsin \frac{r_{K}-r}{x}+r_{K}^{2} \arcsin \frac{r_{K}}{R-x}+r^{2} \arcsin \frac{r}{R}, \\
\frac{1}{2} p(x)= & \sqrt{x^{2}-\left(r_{K}-r\right)^{2}}+\sqrt{(R-x)^{2}-r_{K}^{2}}+\sqrt{R^{2}-r^{2}} \\
& +\left(r_{K}-r\right) \arcsin \frac{r_{K}-r}{x}+r_{K} \arcsin \frac{r_{K}}{R-x}+r \arcsin \frac{r}{R} .
\end{aligned}
$$

So, we have just to study these functions and to obtain their minimum. It can be checked that the first derivatives are

$$
\begin{aligned}
A^{\prime}(x) & =-r_{K} \sqrt{1-\left(\frac{r_{K}}{R-x}\right)^{2}}+\left(r_{K}+r\right) \sqrt{1-\left(\frac{r_{K}-r}{x}\right)^{2}}, \\
\frac{1}{2} p^{\prime}(x) & =-\sqrt{1-\left(\frac{r_{K}}{R-x}\right)^{2}}+\sqrt{1-\left(\frac{r_{K}-r}{x}\right)^{2}} .
\end{aligned}
$$

But it always hold

$$
\sqrt{1-\left(\frac{r_{K}-r}{x}\right)^{2}} \geq \sqrt{1-\left(\frac{r_{K}}{R-x}\right)^{2}}
$$

in fact, inequality (3.6) is equivalent to the easier $\left(r_{K}-r\right) / x \leq r_{K} /(R-x)$, which is, in turn, equivalent to the relation

$$
x \geq \frac{R\left(r_{K}-r\right)}{2 r_{K}-r} ;
$$

but since it holds the lower bound in (3.5), and also $r_{K}>r$, we can obtain easily (3.7) and hence, (3.6). 
All in all, inequality (3.6) assures that both derivatives $A^{\prime}(x)$ and $\frac{1}{2} p^{\prime}(x)$ are (strictly) positive, and hence, that $A(x)$ and $(1 / 2) p(x)$ are (strictly) increasing functions on the interval $\left[R\left(r_{K}-r\right) / r, R-r_{K}\right]$. It proves the required result:

$$
\begin{aligned}
A \geq A\left(\frac{R}{r}\left(r_{K}-r\right)\right) & =\frac{r_{K}^{2}}{r}\left(\sqrt{R^{2}-r^{2}}+\frac{1}{r_{K}} \sqrt{R^{2}\left(2 r-r_{K}\right)^{2}-r^{2} r_{K}^{2}}\right)+r_{K}^{2} \theta, \\
\frac{1}{2} p \geq p\left(\frac{R}{r}\left(r_{K}-r\right)\right) & =\frac{r_{K}}{r} \sqrt{R^{2}-r^{2}}+\frac{1}{r} \sqrt{R^{2}\left(2 r-r_{K}\right)^{2}-r^{2} r_{K}^{2}}+r_{K} \theta,
\end{aligned}
$$

where $\theta=\arcsin \frac{r r_{K}}{R\left(2 r-r_{K}\right)}+\arcsin \frac{r}{R}$.

The equality holds, in both inequalities, if and only if $x=R\left(r_{K}-r\right) / r$, i.e., when the common support lines to $c_{r}$ and $c_{K}$ intersect on $N$. Therefore, the extremal set is the one described in the statement of the theorem: the convex hull conv $\left\{K_{d}, M\right\}$, where $M \in \partial C_{R}$ is the diametrically opposite point to $N$ (see figure $8(\mathrm{~d})$ ).

\section{Optimizing the diameter}

In this section we are going to state the relation among the minimal annulus, the inradius and the diameter of a convex body $K$. The upper bound is almost trivial:

Proposition 2. Let $K$ be a convex body with minimal annulus $A(c, r, R)$ and inradius $r_{K}$. Then:

$$
D \leq 2 R
$$

where equality holds for any set containing diametrically opposite points of $\partial C_{R}$; for instance, the convex body $K^{2 c}=\operatorname{conv}\left\{c_{r} \cup c_{K}, N, M\right\}$, where $\{N, M\}=c y_{0} \cap \partial C_{R}$ (see figure 9 ).

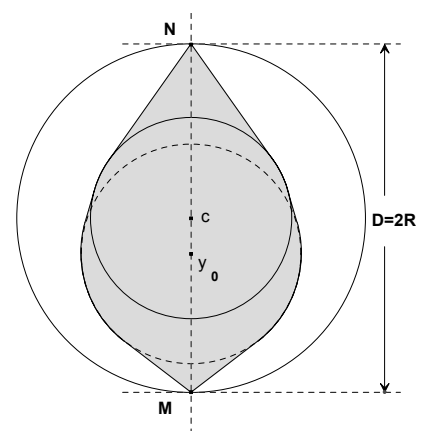

Figure 9: The convex body $K^{2 c}$ has maximum diameter. 
Proof: Inequality (4.1) holds trivially, independently of the value of the inradius, since $K \subset C_{R}$. Now, the set described in the statement of the proposition has minimal annulus $A(c, r, R)$, inradius $r_{K}$, and diameter exactly $D\left(K^{2 c}\right)=d(N, M)=2 R$.

Theorem 2. Let $K$ be a convex body with minimal annulus $A(c, r, R)$ and inradius $r_{K}$. Then:

$$
D \geq \begin{cases}\frac{r_{K}}{r}(R+r) & \text { if } r_{K} \geq 2 r \sqrt{\frac{R-r}{R+r}}, \\ 2 \sqrt{R^{2}-r^{2}} & \text { if } r_{K} \leq 2 r \sqrt{\frac{R-r}{R+r}} .\end{cases}
$$

The equality holds in both cases, for instance, for the set $\operatorname{conv}\left\{K_{d}, M\right\}$, where $M$ is the second intersection point (besides $N$ ) of any of the two common support lines to $c_{r}$ and $c_{K}$ with $\partial C_{R}$ (see figure 10).

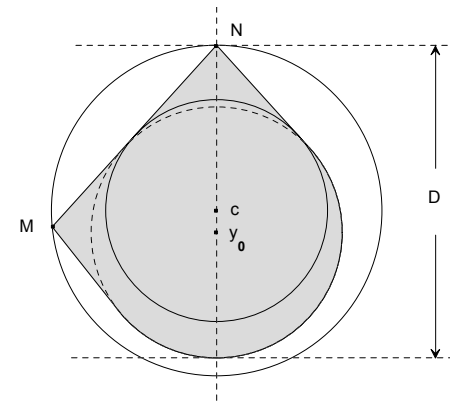

(a)

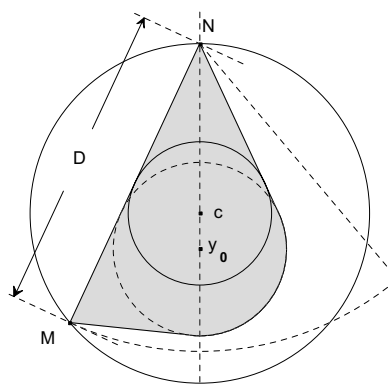

(b)

Figure 10: The convex body $\operatorname{conv}\left\{K_{d}, M\right\}$ has minimum diameter.

Proof: Let us note that if $r_{K}=r$, the relation $r_{K} \geq 2 r \sqrt{(R-r) /(R+r)}$ is equivalent to $R \leq 5 \mathrm{r} / 3$. Hence, inequalities (4.2.a) and (4.2.b) turn into

$$
D \geq \begin{cases}R+r & \text { if } R \leq 5 r / 3 \\ 2 \sqrt{R^{2}-r^{2}} & \text { if } R \geq 5 r / 3\end{cases}
$$

respectively, which are known (see [6, Prop. 3]); in both cases, the extremal sets are also transformed in the corresponding ones: 2-cap-bodies such that the line segment determined by the two vertices (lying on $\partial C_{R}$ ) is tangent to $\partial c_{r}$. Thus, from now on we will assume that $r_{K}>r$ and hence, that $c_{K} \not \equiv c_{r}$.

Lemma 2(vi) assures that $K$ contains a set $K^{2 c}=\operatorname{conv}\left\{c_{r} \cup c_{K}, N, M\right\}$, for suitable $N, M \in \partial C_{R}$, with the same minimal annulus and inradius as $K$. 
Hence, $D \geq D\left(K^{2 c}\right)$, and it suffices to study the diameter for this particular family of sets. But because of the shape of $K^{2 c}$, it is clear that its diameter is attained in one of the following distances:

$\left(d_{1}\right)$ distance between $N$ and $M$;

$\left(d_{2}\right)$ distance between $N$ and the tangent line to $\partial c_{K}$ orthogonal to $N y_{0}$;

$\left(d_{3}\right)$ distance between $M$ and the tangent line to $\partial c_{K}$ orthogonal to $M y_{0}$;

$\left(d_{4}\right)$ distance between $M$ and the tangent line to $\partial c_{r}$ orthogonal to $M c$.

Let us notice that, since $r_{K}>r$, the last two possibilities are not feasible, since both $d_{3}$ and $d_{4}$ give values less than the one of the second option, $d_{2}$. So, we have to study just the above first two distances: $d_{1}$ and $d_{2}$.

The smallest possible distance between $N$ and $M$ (see lemma 1(a)) is attained when the line segment $\overline{M N}$ is tangent to $\partial c_{r}$, and hence, when the straight line $N M$ coincides with one of the common support lines to $c_{r}$ and $c_{K}$. This distance is always $d_{1}=2 \sqrt{R^{2}-r^{2}}$, independently of the situation of the circles $c_{r}$ and $c_{K}$.

On the other hand, the distance $d_{2}$ is less as closer from $c$ lies $y_{0}$, attaining the minimum in the limit case when $d\left(y_{0}, c\right)=R\left(r_{K}-r\right) / r$; i.e., when the common support lines to $c_{r}$ and $c_{K}$ intersect on $N$. The value of such a distance is $r_{K}(R+r) / r$ (see figure 11).

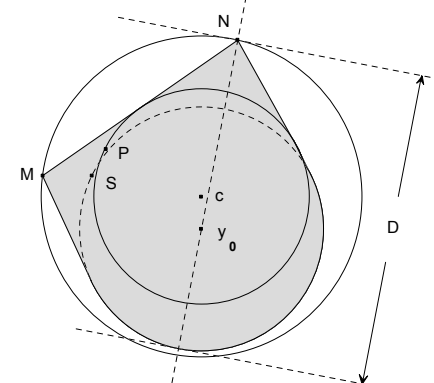

(a)

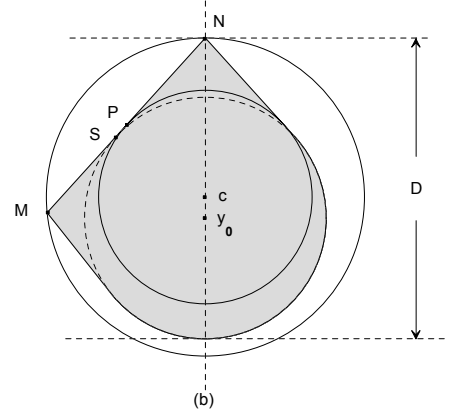

(b)

Figure 11: The distance $d_{2}$ is minimal if $d\left(y_{0}, c\right)=R\left(r_{K}-r\right) / r$.

In summary, the diameter will take either the value $2 \sqrt{R^{2}-r^{2}}$, when it is attained in the distance $d_{1}=d(N, M)$, or $r_{K}(R+r) / r$, if it is attained in the distance $d_{2}$ from $N$ to the support line to $c_{K}$ orthogonal to $N y_{0}$; it depends on the relation between $R, r$ and $r_{K}$. It is easy to check that $D \geq r_{K}(R+r) / r$ if $r_{K} \geq 2 r \sqrt{(R-r) /(R+r)}$ (see figure 10(a)), and that $D \geq 2 \sqrt{R^{2}-r^{2}}$ when $r_{K} \leq 2 r \sqrt{(R-r) /(R+r)}$ (see figure 10(b)). 


\section{Optimizing the minimal width}

In this section we state the relation between the minimal annulus, the inradius and the minimal width of a convex body $K$. The lower bound is almost trivial:

Proposition 3. Let $K$ be a convex body with minimal annulus $A(c, r, R)$ and inradius $r_{K}$. Then:

$$
\omega \geq 2 r_{K},
$$

where equality holds for any set containing in its boundary diametrically opposite points of $\partial c_{K}$; for instance, the convex body $K^{2 c}=\operatorname{conv}\left\{c_{r} \cup c_{K}, N, M\right\}$, where $\{N, M\}=c y_{0} \cap \partial C_{R}$ (see figure 12).

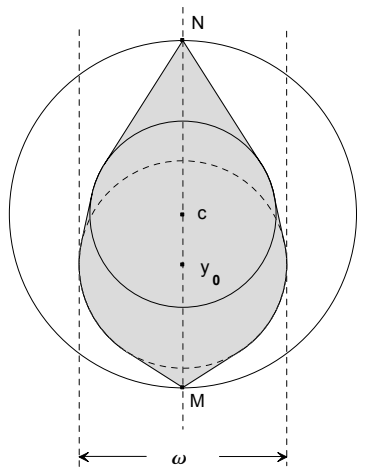

Figure 12: The convex body $K^{2 c}$ has minimum width.

Proof: Inequality (5.1) holds trivially, independently of the minimal annulus, since $K \supset c_{K}$. Now, the set described in the statement of the proposition has minimal annulus $A(c, r, R)$, inradius $r_{K}$, and minimal width exactly $\omega\left(K^{2 c}\right)=2 r_{K}$.

Theorem 3. Let $K$ be a convex body with minimal annulus $A(c, r, R)$ and inradius $r_{K}$. Then:

$$
\omega \leq \begin{cases}R+r & \text { if } R \leq 2 r, \\ \frac{4 r}{R^{2}}\left(R^{2}-r^{2}\right) & \text { if } R \geq 2 r \text { and } r_{K} \geq \frac{2 r(R-r)}{R}, \\ \frac{4 r}{R^{2}}\left(R^{2}-r^{2}\right) \frac{\sin \beta}{\sin (\alpha+\beta)} & \text { if } R \geq 2 r \text { and } r_{K} \leq \frac{2 r(R-r)}{R},\end{cases}
$$

where

$$
\alpha=2 \arcsin \frac{r}{R} \quad \text { and } \quad \beta=2 \arctan \frac{r r_{K}}{\left(2 r-r_{K}\right) \sqrt{R^{2}-r^{2}}} .
$$


The equality holds, in inequalities (5.2.a) and (5.2.b), for instance, for the convex bodies $K^{B}$ shown in figures 13(a,b): let us consider the circular slice of $C_{R}$ determined by the common support lines to $c_{r}$ and $c_{K}, \ell^{\prime}$ and $\ell^{\prime \prime}$, when they intersect on the point $N \in \partial C_{R}$; and let us take the halfplane delimited by the support line to $c_{K}$, orthogonal to $c y_{0}$, containing $c_{K}$; the intersection of both sets gives $K^{B}$.

In inequality (5.2.c), the equality holds only for the triangle determined by $\ell^{\prime}, \ell^{\prime \prime}$, and the support line to $c_{K}$ passing through the point $M^{\prime} \in \ell^{\prime} \cap \partial C_{R}$, $M^{\prime} \neq N$ (see figure 13(c)).

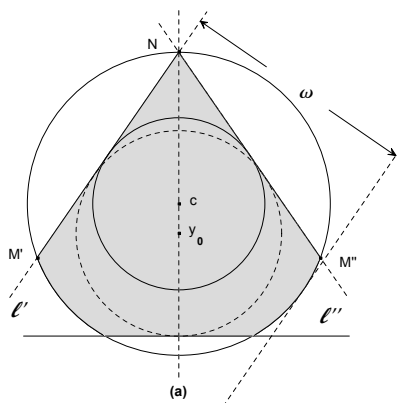

Figure 13: The convex bodies with maximum minimal width.

Let us note that the triangle obtained as extremal set of inequality (5.2.c) verifies that its angles $\alpha$ and $\beta$ (as previously defined, see figure 13 (c)) are always less or equal than the third one, $\pi-\alpha-\beta$. It implies, in particular, that $2 \beta+\alpha \leq \pi$, i.e., $\alpha+\beta \leq \pi-\beta$. Consequently, $\sin \beta \leq \sin (\alpha+\beta)$, which assures that the upper bound in (5.2.c) is always less (or equal) than the upper bound in (5.2.b), as was to be expected. And they will be equal precisely if $\beta=\pi-\alpha-\beta$, i.e., when the triangle is isosceles, which holds only if $r_{K}=2 r(R-r) / R$.

Proof: When $R \leq 2 r$, it is known that inequality (5.2.a) always holds (for given minimal annulus), independently of the value of the inradius (see [6, Subsect. 3.2, Prop. 2]); hence, we just have to find a convex body with inradius $r_{K}$ verifying the equality. Thus, if we consider the convex body $K^{B}$ defined in the statement of the theorem, it is clear that it has minimal annulus $A(c, r, R)$ and inradius $r_{K}$ (in the particular case $r_{K}=r$, it is enough to consider $c_{K} \equiv c_{r}$ and $\ell^{\prime}, \ell^{\prime \prime}$ to be the support lines to $c_{r}$ which intersect on $\left.N \in \partial C_{R}\right)$. Let us denote by $M^{\prime}$ and $M^{\prime \prime}$ the intersection points of, respectively, $\ell^{\prime}$ and $\ell^{\prime \prime}$ with $\partial C_{R}$ (different from $N$, see figure 13 (a)). Let us note that, if $R=2 r$, then the triangle $N M^{\prime} M^{\prime \prime}$ is equilateral and circumscribes $c_{r}$; and since $r_{K} \geq r$, the minimal width of $K^{B}$ is precisely the distance $d\left(M^{\prime \prime}, \ell^{\prime}\right)$. Therefore, if $R \leq 2 r$, the minimal width of $K^{B}$ is attained in the distance between the straight line $\ell^{\prime}$ and its parallel one 
supporting $K^{B}$; and this touching point will lie on the circular arc of $\partial C_{R} \cap$ $\partial K^{B}$ starting in $M^{\prime \prime}$ (see figure 13(a)). This distance is, clearly, $R+r$, which proves the case.

Now we suppose that $R \geq 2 r$. Then, inequality (5.2.b) always holds, independently of the value of the inradius (see [6, Subsect. 3.2, Prop. 2]). Let us state the range of $r_{K}$ for which (5.2.b) keeps its validity. In order to do that, we consider again the set $K^{B}$ previously defined. Let us notice that, if $r_{K}=r$, the relation $r \geq 2 r(R-r) / R$ would be equivalent to $R \leq 2 r$, and hence, inequality (5.2.b) would be nonsense. Therefore, for this case, $r_{K}>r$, and $c_{K} \not \equiv c_{r}$. If $r_{K}$ is large enough for the line segment $\overline{M^{\prime} M^{\prime \prime}}$ intersects $\partial c_{K}$ (see figure 13(b)), then its width is attained in the distance $d\left(M^{\prime \prime}, \ell^{\prime}\right)$, i.e., $4 r\left(R^{2}-r^{2}\right) / R^{2}$. It is an easy computation to check that it happens when $r_{K} \geq 2 r(R-r) / R$ (if $r_{K}=2 r(R-r) / R, \overline{M^{\prime} M^{\prime \prime}}$ just touches $\left.\partial c_{K}\right)$. This proves inequality (5.2.b).

Finally, let us suppose that $R \geq 2 r$ and $r_{K} \leq 2 r(R-r) / R$. Under these assumptions, the line segment $\overline{M^{\prime} M^{\prime \prime}}$ will never intersect $c_{K}$; at most, it will touch its boundary precisely when $r_{K}=2 r(R-r) / R$ (see figure 14).

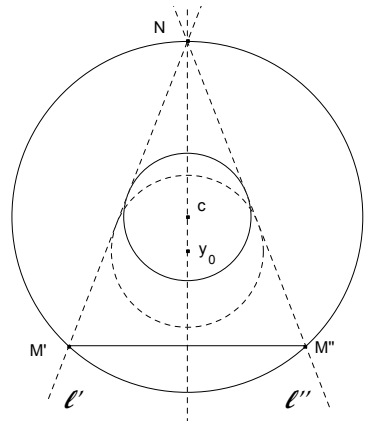

FiguRE 14. If $R \geq 2 r$ and $r_{K} \leq 2 r(R-r) / R$, the line segment $\overline{M^{\prime} M^{\prime \prime}}$ never intersects the incircle $c_{K}$.

Lemma 1(c) states that $K$ is contained in a circular slice $K^{s}$ of $C_{R}$, determined by two support lines to $c_{r}$, and with minimal annulus $A(c, r, R)$; of course, since $K \subset K^{s}$, both $\omega(K) \leq \omega\left(K^{s}\right)$ and $r_{K} \leq r_{K^{s}}$. On the other hand, since $c_{K}$ is the incircle of $K, \partial K$ contains two diametrically opposite points of $\partial c_{K}$, or three points $X, Y, Z \in \partial c_{K}$ forming the vertices of an acuteangled triangle. In the first case, $\omega=2 r_{K}$, which can be excluded, because it gives the minimum value of the width, and we want to maximize it. So, we suppose the existence of $X, Y, Z \in \partial c_{K}$ under the above conditions, which also implies the uniqueness of the incircle.

Let us start assuming that $r_{K}>r$, and consequently, that $c_{K} \not \equiv c_{r}$. Lemma 2(ii) assures that $\operatorname{conv}\left(c_{r} \cup c_{K}\right) \subset K$; hence, the support lines determining $K^{s}$ touch $\partial c_{r}$ in the circular arc $\overparen{P Q} \subset \partial c_{r} \subset \operatorname{conv}\left(c_{r} \cup c_{K}\right)$, whereas the points $X, Y, Z$ lie on the circular $\operatorname{arc} \overparen{S T} \subset \partial c_{K} \subset \operatorname{conv}\left(c_{r} \cup c_{K}\right)$. 
From now on, we are going to use a prime, ${ }^{\prime}$, for denoting the symmetral of a point of $\partial c_{K}$ with respect to $y_{0}$. Following this notation, we can assure that one of the points $X, Y, Z$ lies on the circular arc, either $\overparen{S T^{\prime}}$, or $\overparen{S^{\prime} T}$ : in the opposite case, the three of them would lie on the $\overparen{S^{\prime} T^{\prime}}$, and thus, on a half-circumference, which is impossible (see figure 15(a)). Without loss of generality, let us suppose that $X \in \overparen{S T^{\prime}}$. Then, one of the two remaining points, say $Y$, lies on the circular arc $\overparen{T X^{\prime}}$ (again because otherwise, the three of them would be located on the half-circumference $\overparen{X X^{\prime}}$ ). Finally, since $X, Y, Z$ determine an acute-angled triangle, $Z \in \overparen{X^{\prime} Y^{\prime}} \in \partial c_{K}$. Besides, $K$ is contained in the intersection set of $K^{s}$ with the triangle $T_{X Y Z}$ determined by the support lines $\ell_{X}, \ell_{Y}, \ell_{Z}$, to $c_{K}$ through $X, Y, Z$, respectively (see figure 15).

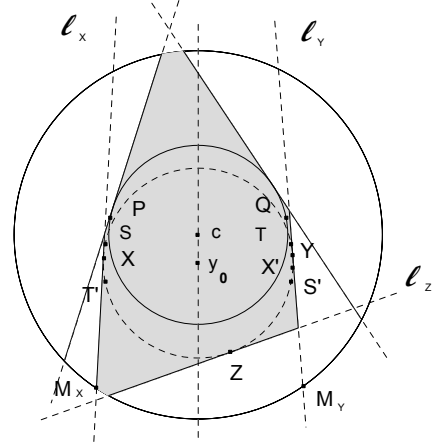

(a)

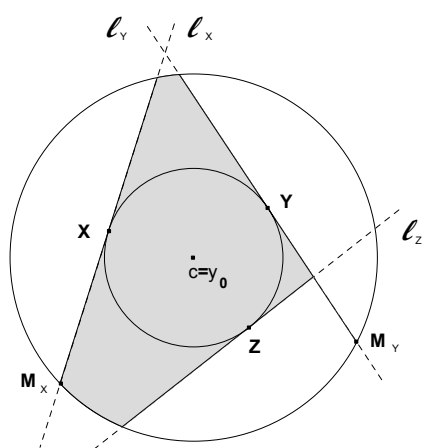

(b)

FiguRE 15: The problem is reduced to study the sets $K^{s} \cap T_{X Y Z}$.

On the other hand, if $r_{K}=r$, then $c_{K} \equiv c_{r}$; in the opposite case, it would be impossible to find three points of $\partial c_{K} \cap \partial K$ determining an acute-angled triangle. Now, there is no arcs $\overparen{P Q}$ or $\overparen{S T}$, because $\operatorname{conv}\left(c_{r} \cup c_{K}\right) \equiv c_{K}$. So, in this case, we will just suppose that $Z \in \overparen{X Y}$ and that lies "below" both points, $X$ and $Y$ (as shown in figure 15(b)). Besides, the set $\bar{K}$ will be just the intersection $\bar{K}=T_{X Y Z} \cap C_{R}$. In the following, almost all the arguments will be valid in both cases, $c_{K} \equiv c_{r}$ or not. So, the remainder part of the proof is the same, and we will distinguish the cases just in the precise moment when it is necessary.

Let us notice that the straight line $\ell_{Z}$ intersects the circular arc $\overparen{M_{X} M_{Y}}$, where $M_{X}, M_{Y} \in \partial K^{s}$ denote the intersection points of $\ell_{X}$ and $\ell_{Y}$ with $\partial C_{R}$ : otherwise, there would be no point of $\partial C_{R} \cap \partial K$ on the arc $\overparen{M_{X} M_{Y}}$, which would be a contradiction to property (P2) $\left(\partial C_{R} \cap \partial K\right.$ and $\partial c_{r} \cap \partial K$ could be separated). 
It is clear that the set $K^{\prime}$ has minimal annulus $A(c, r, R)$ and inradius $r_{K}$. Since $K \subset \bar{K}, \omega(K) \leq \omega(\bar{K})$, and the problem has been reduced to study the width of the convex bodies belonging to this particular family. Let us note the following:

1. The angle determined by $\ell_{X}$ and $\ell_{Y}$ is always less or equal than the one corresponding to the straight lines that determine $K^{s}$ (when $r_{K}>r$; if $r_{K}=r, K^{s}$ plays no role, and this observation is not considered).

2. The angle determined by $\ell_{X}$ and $\ell_{Y}$ can not vanish, i.e., $\ell_{X}$ and $\ell_{Y}$ will never be parallel, since in this case the points $X, Y, Z$ would lie on the same half-circumference.

3. Since $X, Y, Z$ are not on a half-circumference, the intersection point $\ell_{X} \cap \ell_{Y}$ lies on the upper halfplane determined by the orthogonal line to $c y_{0}$ passing through $c$; besides, $\ell_{X} \cap \ell_{Y}$ is not an interior point of the circle $C_{R}$.

4. The triangle $T_{X Y Z}$ has inradius $r_{K}$ and verifies that, at most, just one of its vertices (either $\ell_{Y} \cap \ell_{Z}$ or $\ell_{X} \cap \ell_{z}$ ) lies in the interior of $C_{R}$.

From these observations we can conclude that the minimal width of $\bar{K}$ will be attained in the distance of the only vertex of $T_{X Y Z}$ which lies in the interior of $C_{R}$ to the opposite side, if it exists, or it will be, in the opposite case, the minimum of the distances $d\left(M_{X}, \ell_{Y}\right), d\left(M_{Y}, \ell_{X}\right)$. But in any of these two cases, the minimal width will increase, as bigger is the angle $\theta$ determined by the lines $\ell_{X}$ and $\ell_{Y}$ : in fact, both the only "interior" vertex of $T_{X Y Z}$ (if it exists), as well as the points $M_{X}, M_{Y}$ increase their distance to their corresponding opposite sides (either $\ell_{X}$ or $\ell_{Y}$ ) when $\theta$ increases.

Thus, let us first note that, if $r_{K}>r$ and we fix $c_{K}$, the angle $\theta$ will be maximal when the points $X$ and $Y$ coincide, respectively, with the extreme points $S$ and $T$ of the circular arc of $\partial c_{K} \subset \partial\left(c_{r} \cup c_{K}\right)$. Now, after this observation, and thus assuming that $X \equiv S$ and $Y \equiv T$, it is clear that $\theta$ will also increase (and hence the minimal width) as closer is the incenter $y_{0}$ to $c$ (see figure 16). The limit case will be when $d\left(y_{0}, c\right)=R\left(r_{K}-r\right) / r$, i.e., when the lines $\ell_{X}, \ell_{Y}$ are, precisely, the common support lines, $\ell^{\prime}, \ell^{\prime}$, to $c_{r}$ and $c_{K}$ which intersect on $N \in \partial C_{R}$.

Let us notice that if $r_{K}=r$, the above argument is trivial: we just have to increase the angle $\theta$ till $\ell_{X} \cap \ell_{Y} \in \partial C_{R}$, because $y_{0} \equiv c$.

On the other hand, for any possible position of the incircle $c_{K}$ (even, in the case $c_{K} \equiv c_{r}$ ), and fixing the lines $\ell_{X}$ and $\ell_{Y}$, it is clear that the minimal width of $\bar{K}$ is larger as closer is $Z$ to the intersection point $c y_{0} \cap \partial c_{K}$ $\left(N y_{0} \cap \partial c_{K}\right.$ if $\left.c \equiv y_{0}\right)$. But let us recall that we are assuming $R \geq 2 r$ and $r_{K} \leq 2 r(R-r) R$, which implies that, in the limit situation for the maximum 
minimal width (i.e., $\ell_{X} \equiv \ell^{\prime}, \ell_{Y} \equiv \ell^{\prime \prime}$ and $\ell_{X} \cap \ell_{Y}=N \in \partial C_{R}$ ), the line segment $\overline{M_{X} M_{Y}} \equiv \overline{M^{\prime} M^{\prime \prime}}$ does not intersect $c_{K}$; at most it will touch $\partial c_{K}$ when $R=2 r$ and $r_{K}=r$. Hence, the real limit position for the point $Z$ is the one when $\ell_{Z}$ passes, precisely, through $M_{X}$ (or equivalently $M_{Y}$, because of the symmetry of the figure), see figure 16).

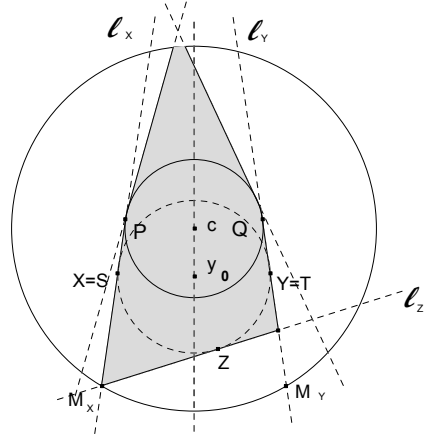

(a)

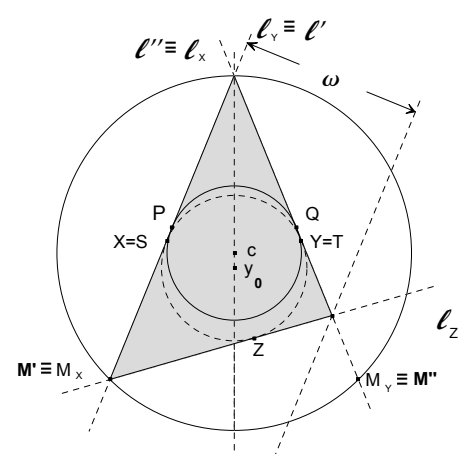

(b)

FiguRE 16: Moving the incenter in order to increase the minimal width.

In this way, we can conclude that under the assumptions of the theorem, the convex body with maximum minimal width is the triangle determined by the straight lines $\ell^{\prime}, \ell^{\prime \prime}$, and the support line to $c_{K}$ passing through $M^{\prime}$ (see figure 13). An easy computation shows that the minimal width of this triangle is given by

$$
\omega=2 \sqrt{R^{2}-r^{2}} \frac{\sin \alpha \sin \beta}{\sin (\alpha+\beta)}=\frac{4 r}{R^{2}}\left(R^{2}-r^{2}\right) \frac{\sin \beta}{\sin (\alpha+\beta)},
$$

where

$$
\alpha=2 \arcsin \frac{r}{R} \quad \text { and } \quad \beta=2 \arctan \frac{r r_{K}}{\left(2 r-r_{K}\right) \sqrt{R^{2}-r^{2}}}
$$

which proves inequality (5.2.c).

\section{References}

[1] BÁRÁNy, I.: On the minimal ring containing the boundary of a convex body. Acta Sci. Math. (Szeged) 52 (1988), no. 1-2, 93-100.

[2] Bonnesen, T.: Les problèmes des isopérimètres et des isépiphanes. Collection de monographies sur la théorie des fonctions. Gauthier-Villars, Paris, 1929.

[3] Bonnesen, T. And Fenchel, W.: Theorie der konvexen Körper. Springer, Berlin 1934, 1974; Chelsea, New York, 1948. 
[4] Favard, J.: Problèmes d'extremums relatifs aux courbes convexes. I, II. Ann. Sci. École Norm. Sup. (3) 46-47 (1929-30), 345-369, 311-324.

[5] Favard, J.: Sur le déficit isopérimétrique maximum dans une couronne circulaire. Mat. Tidsskr. B (1929), 62-68.

[6] Hernández Cifre, M. A. and Herrero, P.: Some optimization problems for the minimal annulus of a convex set. Math. Inequal. Appl. 9 (2006), 359-374.

[7] Hernández Cifre, M. A. and Herrero, P.: Relating the minimal annulus with the circumradius of a convex set. Submitted, 2007.

[8] Peri, C.: On the minimal convex shell of a convex body. Canad. Math. Bull. 36 (1993), no. 4, 466-472.

[9] Peri, C.: Minimal shells containing a convex surface in Minkowski space. Manuscripta Math. 90 (1996), no. 3, 333-342.

[10] Peri, C. And Vassallo, S.: Minimal properties for convex annuli of plane convex curves. Arch. Math. (Basel) 64 (1995), no. 3, 254-263.

[11] Peri, C. And Zucco, A.: On the minimal convex annulus of a planar convex body. Monatsh. Math. 114 (1992), no. 2, 125-133.

[12] Yaglom, I. M. and Boltyanskĭ, V. G.: Convex figures. Holt, Rinehart and Winston, Russian original, Moscow-Leningrad 1951; New York, 1961.

[13] Zucco, A.: Minimal annulus of a convex body. Arch. Math. (Basel) 52 (1989), no. 1, 92-94.

[14] Zucco, A.: Minimal shell of a typical convex body. Proc. Amer. Math. Soc. 109 (1990), no. 3, 797-802.

Recibido: 2 de marzo de 2006

Revisado: 19 de mayo de 2006

María A. Hernández Cifre Departamento de Matemáticas

Universidad de Murcia 30100-Murcia, Spain mhcifre@um.es

Pedro J. Herrero Piñeyro Departamento de Matemáticas

Universidad de Murcia 30100-Murcia, Spain pherrero@um.es

This work is supported in part by Dirección General de Investigación (MEC) MTM200764504 and by Fundación Séneca (C.A.R.M.) 00625/PI/04. 